\title{
Trajectory Analysis and Predictors of the Percentage of Body Fat Among Chinese Sleeve Gastrectomy Patients
}

\author{
Kang Zhao $\mathbb{D}^{\prime}$ \\ Xinyi $X u^{1,2}$ \\ Hanfei Zhu' \\ Ziqi Ren ${ }^{3}$ \\ Tianzi Zhang ${ }^{4}$ \\ Ningli Yang ${ }^{5}$ \\ Shuqin Zhu' \\ Qin $X u^{\prime}$
}

'School of Nursing, Nanjing Medical University, Nanjing, Jiangsu, People's Republic of China; ${ }^{2}$ Faculty of Health, The Queensland University of Technology, Brisbane, Queensland, Australia; ${ }^{3}$ School of Nursing, Fudan University, Shanghai, People's Republic of China; ${ }^{4}$ Department of Nursing, Jiangsu College of Nursing, Huai'an, Jiangsu, People's Republic of

China; ${ }^{5}$ Department of General Surgery, The First Affiliated Hospital of Nanjing Medical University, Nanjing, Jiangsu, People's Republic of China
Correspondence: Qin Xu; Shuqin Zhu Tel +86 I360I587208; +86 I385I9464I3 Email qinxu@njmu.edu.cn; zsq@njmu.edu.cn
Background: The weight loss in Chinese patients after sleeve gastrectomy is different, and the differences can be evaluated through the trajectories of the percentage of body fat $(\mathrm{BF} \%)$. Patients' baseline psychosocial factors may be associated with these trajectories.

Materials and Methods: We selected 267 patients who received sleeve gastrectomy for the first time. The BF\% at baseline and 1, 3, 6, 12 months after surgery and baseline psychosocial variables were retrospectively collected. The trajectory model was established according to $\mathrm{BF} \%$ based on the growth mixture model. The baseline psychosocial variables were compared among different trajectory classes.

Results: Four types of trajectory classes were obtained. The differences in preoperative dietary self-efficacy, exercise self-efficacy, depression, social support, working status, alcohol consumption, and gender among the classes were statistically significant. The pairwise comparison of the above variables revealed that the differences of gender, dietary selfefficacy and exercise self-efficacy among classes were highly effective.

Conclusion: Female gender, low dietary self-efficacy and low exercise self-efficacy were predictors for poor $\mathrm{BF} \%$ trajectory in sleeve gastrectomy patients. Health professionals can early identify patients who are most likely to lose weight in a not-ideal manner based on the above predictors.

Keywords: bariatric surgery, body fat, psychosocial aspects, sleeve gastrectomy, trajectory

\section{Introduction}

Obesity is considered an epidemic in the 21 st century, accounting for 4.72 million deaths and 148 million disability-adjusted life-years globally. ${ }^{1}$ Sleeve gastrectomy (SG) is one of the most relevant bariatric procedures for treating severely obese patients in China. ${ }^{2}$ Patients usually have to gradually switch from a liquid diet to a light, highprotein diet for 3 months after surgery, and should continue to supplement vitamins and trace elements in order to consolidate the effect of the surgery. As regards the physical activity, patients should perform aerobic exercise for at least 150 minutes per week. However, the weight loss varies among patients ${ }^{3,4}$ because of their different behavior and lifestyle, ${ }^{5}$ which is mainly affected by their psychosocial factors. ${ }^{6,7}$ Therefore, the psychosocial factors can be considered as potential predictors of not-ideal weight loss outcomes.

In terms of patients' weight loss outcomes, the percentage of body fat $(\mathrm{BF} \%)$ was considered as an indicator, ${ }^{8}$ which represents the proportion of body fat weight per total weight. As regard the post-bariatric patients, the overall changes in body weight may 
mask changes in body composition, thus, patients with the same weight may have different body fat mass. $\mathrm{BF} \%$ is the key factor determining the risk of cardiovascular and metabolic diseases. ${ }^{9}$ Patients with high BF\% after bariatric surgery show low insulin sensitivity and high central fat distribution independent of $\mathrm{BMI} .{ }^{10} \mathrm{~A}$ low $\mathrm{BF} \%$ is more consistent with the surgical goals. Besides, the development of trajectory models is a good method to identify potential different subpopulations within a population, helping to identify SG patients with not-ideal body fat loss effects. ${ }^{11,12}$

Therefore, a $\mathrm{BF} \%$ trajectory model of $\mathrm{SG}$ patients was constructed, and the psychosocial data at baseline were compared among different trajectories, to preoperatively identify which patients could have unexpected body fat loss outcomes to provide a better management of the postoperative behavior.

\section{Materials and Methods}

This work was a retrospective study conducted in the Bariatric Surgery Center of the First Affiliated Hospital of Nanjing Medical University. This study was conducted in compliance with the Declaration of Helsinki.

\section{Research Samples and Data}

This study included patients who first underwent SG between December 2015 and December 2019 and were subjected to the complete measurement of body composition at baseline, and at $1^{\text {st }}, 3^{\text {rd }}, 6^{\text {th }}$, and $12^{\text {th }}$ month after surgery. Patients who did not complete the baseline psychosocial assessment, suffered serious complications (including gastrointestinal bleeding, anastomotic fistula, and stricture), refused to allow the use of their data, or were pregnant, were excluded from this study. Our bariatric center performed 825 SG during these four years, and finally, the data of 267 patients (32.4\%) were retrospectively collected (Supplementary Materials). Figure 1 shows the flowchart of patients' selection. Patients' BF\% was extracted from the body composition analysis instrument (Version: Inbody 770, Seoul, Korea), while the baseline psychosocial data were extracted from the medical records and previous studies. All psychosocial data were evaluated by the case manager on the admission day.

\section{Measures}

\section{Demographic and Clinical Data}

The following data were extracted from the patients' medical records: age, gender, education, marital status, working status, medical insurance, smoking status, alcohol consumption, and preoperative obesity comorbidities (including hypertension, type 2 diabetes, obstructive sleep apnea, and dyslipidemia).

\section{Trajectory Data of BF\%}

The measurements were performed using an 8-point contact multi-frequency impedance method by InBody 770 . During the measurement, participants should bring their thumbs close to each other to handle the electrodes, spread their hands at an angle of $15^{\circ}$, and should be barefoot and place their heels and soles on either side of the electrodes. The measurements included the body weight, $\mathrm{BF} \%$, and the resting metabolic rate. All patients included in this study completed the measurements at the $1^{\text {st }}, 3^{\text {rd }}, 6^{\text {th }}$, and $12^{\text {th }}$ month after surgery. ${ }^{13}$

\section{Scale Evaluation Data}

This study also collected patients' psychosocial data evaluated by 5 different types of scales. (1) Weight efficacy lifestyle questionnaire-short form (WEL-SF) ${ }^{14}$ was used to assess the degree of confidence of the patients in refraining from overeating in different situations. (2) Self-efficacy for exercise (SEE) scale ${ }^{15}$ evaluated patients' confidence in performing exercises in various difficult situations. (3) Patient health questionnaire with 9 items (PHQ-9) ${ }^{16}$ was used to assess patients' depression level. (4) Generalized anxiety disorder 7 -item scale $(\mathrm{GAD}-7)^{17}$ was used to screen the generalized anxiety and the evaluation of symptom severity. (5) Social support rating scale $(\mathrm{SSRS})^{18}$ assessed the level of social support available to patients. All these scales were tested in the Chinese population and revised to obtain the Chinese version, achieving good reliability and validity.

\section{Statistical Analysis}

Statistical analysis was performed using IMB SPSS Statistics version 22.0 (IMB Corp., Armonk, NY, USA). $\mathrm{BF} \%$ trajectory model in 267 patients at five time points was established by M-plus version 8.0 (Muthen\&Muthen, Los Angeles, CA, USA). Growth mixture model (GMM) ${ }^{19}$ was used to identify the subgroups with similar postoperative $\mathrm{BF} \%$ changes. Fitting indexes included Akaike information criterion (AIC), Bayesian information criterion (BIC), entropy, Lo-Mendell-Rubin likelihood ratio test (LMR), bootstrapped likelihood ratio test (BLRT), and category probability. The smaller the value of AIC and BIC, the better the fitting model. Entropy close to 1 implies a precise classification. LMR and BLRT values are less than 0.05 , indicating that the model classified into 
Patients who first underwent SG between December 2015 and December $2019(n=825)$
Patients excluded because of could not construct trajectories due to lack of complete $\mathrm{BF} \%$ data $(\mathrm{n}=496)$
Patients subjected to the complete measurement of body composition at baseline, and at $1 \mathrm{st}, 3 \mathrm{rd}$, 6 th, and 12 th month after surgery $(n=329)$

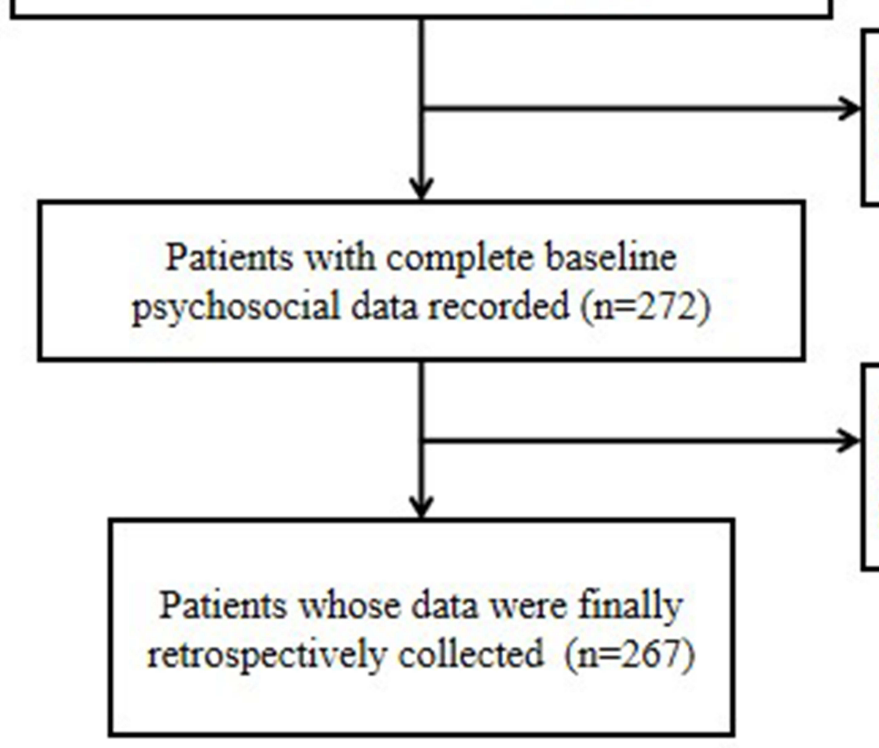

Patients excluded because of incomplete baseline psychosocial data $(n=57)$
Patients excluded due to severe complications $(n=2)$, pregnancy $(n=2)$, and refusal of data to be used $(\mathrm{n}=1)$

Figure I Flow chart of patients' selection. Severe complications include gastrointestinal bleeding, anastomotic fistula, and stricture. Abbreviations: SG, sleeve gastrectomy; BF\%, percentage of body fat.

$\mathrm{k}$ classes is significantly better than $\mathrm{k}-1$ classes. The category probability of each class should be more than $5 \%$. The model was fitted from 2 potential classes, and then fitted according to 3,4 , and 5 potential classes in turn. The most appropriate fitting model was finally selected by comparing the fitting indexes of different trajectory models and referring to the theoretical significance.

Continuous data normally distributed were expressed as mean \pm standard deviation. Counting data were described by frequency. The psychosocial factors of patients with different trajectory classes were compared using the analysis of variance (ANOVA) and Chi-square test. Variables with significant difference were subjected to pairwise comparison, in which the effects of each variable were calculated by Cohen's-d. An absolute value of Cohen's-d $>0.5$ in Student's $t$-test reflected a medium effect and $>0.8$ reflected a high effect. An absolute value of Cohen's-d $>0.3$ in chi-square test reflected a medium effect and $>0.5$ reflected a high effect. A two-sided $p$ value $<0.05$ was considered statistically significant.

\section{Results}

Among the 267 patients, $57.3 \%$ were female $(n=153)$ with an average age of $30.67 \pm 10.28$ years. The average score of WEL-SF, SEE, PHQ-9, GAD-7, and SSES at baseline was $44.57 \pm 14.76, \quad 48.30 \pm 17.22,4.30 \pm 3.05,2.65 \pm 1.96$, and $31.00 \pm 7.80$, respectively. The details of the baseline sociodemographic characteristics and disease-related data are shown in Table 1.

Table 2 shows the fitting results of the candidate models with different classes. When the classes were 2, 3 and 4, the LMR and BLRT tests were statistically significant. 
Table I Data of Patients at Baseline

\begin{tabular}{|c|c|c|}
\hline Variables & Classifications & Number or Mean \pm Standard Deviation \\
\hline \multirow[t]{2}{*}{ Gender } & Male & 114 \\
\hline & Female & 153 \\
\hline Age(years) & & $30.67 \pm 10.28$ \\
\hline \multirow[t]{4}{*}{ Education level } & Primary school or below & 15 \\
\hline & Middle school & 50 \\
\hline & High school or technical school & 52 \\
\hline & College, undergraduate or above & 150 \\
\hline \multirow[t]{2}{*}{ Marital status } & Married & 133 \\
\hline & Unmarried, divorced or widowed & 134 \\
\hline \multirow[t]{2}{*}{ Working status } & Employed & 163 \\
\hline & Retired, unemployed or at school & 104 \\
\hline \multirow[t]{2}{*}{ Medical insurance } & Yes & 152 \\
\hline & No & 115 \\
\hline \multirow[t]{2}{*}{ Smoking } & Yes & 26 \\
\hline & No or ceased & 241 \\
\hline \multirow[t]{2}{*}{ Alcohol consumption } & Yes & 18 \\
\hline & No or ceased & 249 \\
\hline \multirow[t]{2}{*}{ Comorbidities (hypertension, T2DM, OSA, NAFLD, etc.) } & Yes & 198 \\
\hline & No & 69 \\
\hline Baseline BMI $\left(\mathrm{kg} / \mathrm{m}^{2}\right)$ & & $39.04 \pm 6.86$ \\
\hline Baseline BF\% & & $43.06 \pm 7.13$ \\
\hline WEL-SF score & & $44.57 \pm 14.76$ \\
\hline SEE score & & $48.30 \pm 17.22$ \\
\hline PHQ-9 score & & $4.30 \pm 3.05$ \\
\hline GAD-7 score & & $2.65 \pm 1.96$ \\
\hline SSRS score & & $31.00 \pm 7.81$ \\
\hline
\end{tabular}

Note: Data are presented as number or mean \pm standard deviation.

Abbreviations: T2DM, type 2 diabetes mellitus; OSA, obstructive sleep apnea; NAFLD, non-alcoholic fatty liver disease; BMI, body mass index; WEL-SF, weight efficacy lifestyle questionnaire-short form; SEE, self-efficacy for exercise scale; PHQ-9, patient health questionnaire; GAD-7, generalized anxiety disorder 7-item scale; SSRS, social support rating scale.

Table 2 Results of the Model Fitting

\begin{tabular}{|c|c|c|c|c|c|c|}
\hline Number of Trajectory Classes & AIC & BIC & Entropy & LMR & BLRT & Proportion of Each Class \\
\hline 2 & 8533.171 & 8570.472 & 0.834 & $<0.001 *$ & $<0.001 *$ & $0.623 / 0.371$ \\
\hline 3 & $8 \mid 72.611$ & 8221.102 & 0.815 & $0.016 *$ & $<0.001 *$ & $0.340 / 0.269 / 0.391$ \\
\hline 4 & 7992.052 & 8051.733 & 0.828 & $0.035 *$ & $<0.001 *$ & $0.202 / 0.150 / 0.55 \mathrm{I} / 0.097$ \\
\hline 5 & 7854.729 & 7925.601 & 0.822 & 0.072 & $<0.001 *$ & $0.038 / 0.370 / 0.101 / 0.197 / 0.293$ \\
\hline
\end{tabular}

Note: Bold*: $P<0.05$

Abbreviations: AIC, Akaike information criterion; BIC, Bayesian information criterion; LMR, Lo-Mendell-Rubin likelihood ratio test; BLRT, bootstrapped likelihood ratio test.

However, the LMR test result of the model with 5 classes was 0.072 , indicating that the trajectory model of patients divided into 5 classes according to $\mathrm{BF} \%$ was not significantly better than the one into 4 classes. Thus, the model divided into 5 classes was not considered anymore. The model with 4 classes had smaller AIC and BIC values, entropy was closer to 1 , and the proportion of all 4 classes was more than $5 \%$ compared with the models divided into 2 or 3 classes. Therefore, the model with 4 classes was finally selected for subsequent analysis. Figure 2 shows the trajectories of the model.

Patients BF\% in Class-1 remained low for 12 months and dropped relatively fast, thus, this class was called "Low-baseline Fast-loss". A total of 54 patients belonged to the Class-1. Patients in Class-2 had the highest initial $\mathrm{BF} \%$ and did not reach the optimal $\mathrm{BF} \%$ at 12 months $(23.83 \% \pm 6.90)$, but they lost most of the body fat, thus, Class-2 was named "High-baseline 


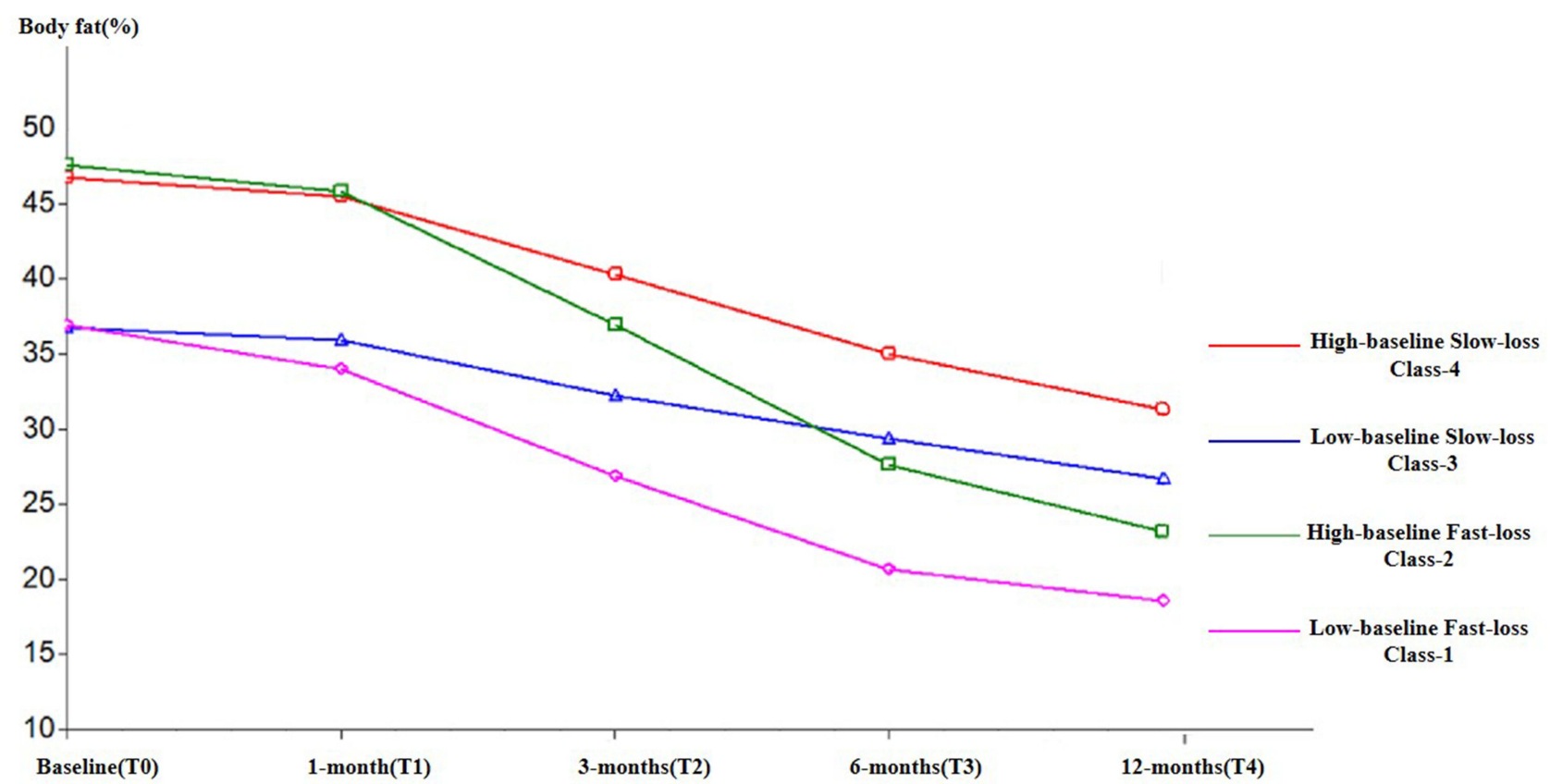

Figure 2 Trajectories of the percentage of body fat. The graph shows the four clusters of loss of patients' percentage of body fat after Sleeve Gastrectomy. The purple trajectory is Class-I (Low-baseline Fast-loss), green one is Class-2 (High-baseline Fast-loss), Blue one is Class-3 (Low-baseline Slow-loss), and Red one is Class-4 (Highbaseline Slow-loss).

Fast-loss" and was composed of 26 patients. In contrast to Class-2, Class-3 had the slowest decrease rate of BF \%. Patients' baseline $\mathrm{BF} \%$ in Class- 3 was not high, but their $\mathrm{BF} \%$ was not ideal at one year after surgery $(27.25 \% \pm 7.22)$. Class-3 was called "Low-baseline Slowloss", and included 40 patients. Patients' BF\% in Class4 decreased slowly and it remained high within 1 year (BF\% 31.85 \pm 3.39 at $12^{\text {th }}$ month). The improvement in body composition that patients obtained after SG was very limited, thus, Class-4 was named "High-baseline Slow-loss", and it included most of the patients $(n=147)$. Although differences in the change of the trends in BF\% were found among different classes of patients, the trajectories showed overall downward trends one year after surgery. The details of each trajectory class are shown in Table 3.
The comparison of the patients' baseline demographic data, body composition data, and psychosocial data revealed a significant difference in gender, working status, alcohol consumption, baseline BF\%, dietary self-efficacy, exercise self-efficacy, depression, and social support among different trajectory classes (Table 4). In addition, no significant difference was observed in baseline BMI despite patients' baseline BF\% widely widely. No significant differences in anxiety, smoking, age and other variables were found among classes.

Next, the significantly different variables were further subjected to pairwise comparison (Table 5). Since BF\% was used to distinguish the trajectory classes, this variable was not compared. No significant difference in the seven variables was found between Class-1 (Low-baseline Fastloss) and Class-2 (High-baseline Fast-loss), as well as

Table 3 Detailed Information of Each Trajectory Class

\begin{tabular}{|l|c|c|c|c|c|}
\hline \multirow{2}{*}{ Trajectory } & \multirow{2}{*}{ Name } & \multicolumn{4}{|c|}{ Detailed Information } \\
\cline { 3 - 6 } & & Baseline BF\% & $1^{\text {th }}$ Month BF\% & $\Delta$ BF\% in I Year & Sample Size \\
\hline Class-1 & Low-baseline Fast-loss & $36.94 \pm 5.00$ & $19.48 \pm 5.34$ & 17.47 & 54 \\
Class-2 & High-baseline Fast-loss & $47.92 \pm 6.79$ & $23.85 \pm 6.90$ & 24.07 & 26 \\
Class-3 & Low-baseline Slow-loss & $36.23 \pm 6.97$ & $27.25 \pm 7.22$ & 8.98 & 40 \\
Class-4 & High-baseline Slow-loss & $46.30 \pm 4.60$ & $31.95 \pm 3.39$ & 14.35 & 147 \\
\hline
\end{tabular}

Abbreviations: $B F \%$, percentage of body fat; $\triangle B F \%$, changes in percentage of body fat. 
Table 4 Comparison of the Baseline Data Among Classes

\begin{tabular}{|c|c|c|c|c|c|c|c|}
\hline \multicolumn{2}{|l|}{ Variables } & \multicolumn{4}{|c|}{ Trajectory Classes } & \multirow[t]{2}{*}{$\chi^{2}$ or $\mathbf{F}$} & \multirow[t]{2}{*}{$\mathbf{P}$} \\
\hline & & \multirow{2}{*}{$\begin{array}{c}\begin{array}{c}\text { Class- } \\
(n=54)\end{array} \\
45\end{array}$} & \multirow{2}{*}{$\begin{array}{l}\text { Class-2 } \\
(n=26)\end{array}$} & \multirow{2}{*}{$\begin{array}{c}\begin{array}{c}\text { Class-3 } \\
n=(40)\end{array} \\
1\end{array}$} & $\begin{array}{l}\text { Class }-4 \\
(n=147)\end{array}$ & & \\
\hline Gender & Male & & & & 37 & 74.082 & $<0.001 *$ \\
\hline & Female & 9 & 5 & 29 & 110 & & \\
\hline Age (years) & & $31.72 \pm 11.72$ & $32.54 \pm 7.70$ & $31.38 \pm 13.42$ & $29.76 \pm 9.08$ & 0.925 & 0.429 \\
\hline \multirow[t]{4}{*}{ Education level } & Primary school or below & 5 & 0 & 4 & 6 & 9.175 & 0.421 \\
\hline & Middle school & 9 & 3 & 6 & 32 & & \\
\hline & High school or technical & 11 & 4 & 6 & 31 & & \\
\hline & $\begin{array}{l}\text { College, undergraduate or } \\
\text { above }\end{array}$ & 29 & 19 & 24 & 78 & & \\
\hline \multirow[t]{2}{*}{ Marital status } & Married & 29 & 17 & 17 & 70 & 3.987 & 0.263 \\
\hline & $\begin{array}{l}\text { Unmarried, divorced or } \\
\text { widowed }\end{array}$ & 25 & 9 & 23 & 77 & & \\
\hline \multirow[t]{2}{*}{ Working status } & Employed & 33 & 21 & 18 & 91 & 8.630 & $0.035 *$ \\
\hline & $\begin{array}{l}\text { Retired, unemployed or at } \\
\text { school }\end{array}$ & 21 & 5 & 22 & 56 & & \\
\hline \multirow[t]{2}{*}{ Medical insurance } & Yes & 31 & 15 & 26 & 80 & 1.451 & 0.694 \\
\hline & No & 23 & 11 & 14 & 67 & & \\
\hline \multirow[t]{2}{*}{ Smoking } & Yes & 6 & 3 & 5 & 12 & 0.974 & 0.808 \\
\hline & No or ceased & 48 & 23 & 35 & 135 & & \\
\hline \multirow[t]{2}{*}{ Alcohol consumption } & Yes & 3 & 0 & 13 & 2 & 50.984 & $<0.001 *$ \\
\hline & No or ceased & 51 & 26 & 27 & 145 & & \\
\hline \multirow[t]{2}{*}{ Comorbidities } & Yes & 38 & 19 & 28 & 113 & 1.345 & 0.718 \\
\hline & No & 16 & 7 & 12 & 34 & & \\
\hline Baseline BMI $\left(\mathrm{kg} / \mathrm{m}^{2}\right)$ & & $37.32 \pm 6.04$ & $39.22 \pm 5.43$ & $38.49 \pm 10.22$ & $39.79 \pm 6.15$ & 1.819 & 0.144 \\
\hline Baseline BF\% & & $36.94 \pm 5.00$ & $47.92 \pm 6.79$ & $36.23 \pm 6.97$ & $46.30 \pm 4.60$ & 71.029 & $<0.00 I^{*}$ \\
\hline WEL-SF score & & $60.33 \pm 13.79$ & $56.27 \pm 11.74$ & $51.83 \pm 13.12$ & $35.87 \pm 16.72$ & 9.346 & $<0.001 *$ \\
\hline SEE score & & $49.35 \pm 15.20$ & $54.38 \pm 13.24$ & $38.90 \pm 15.23$ & $42.68 \pm 13.54$ & 44.183 & $<0.001 *$ \\
\hline PHQ-9 score & & $3.46 \pm 2.31$ & $4.77 \pm 3.78$ & $3.78 \pm 2.57$ & $4.67 \pm 3.21$ & $2.74 I$ & $0.044 *$ \\
\hline GAD-7 score & & $2.13 \pm 1.54$ & $2.92 \pm 1.74$ & $2.40 \pm 1.98$ & $2.86 \pm 2.10$ & 2.264 & $0.08 I$ \\
\hline SSRS score & & $33.09 \pm 9.62$ & $34.08 \pm 9.30$ & $31.83 \pm 7.56$ & $29.46 \pm 6.43$ & 4.901 & $0.002 *$ \\
\hline
\end{tabular}

Notes: Categorical variables were compared by Pearson chi-square test and represented by $\chi^{2}$ value, continuous variables were compared by analysis of variance and represented by $F$ value. Bold*: $P<0.05$.

Abbreviations: BMI, body mass index; WEL-SF, weight efficacy lifestyle questionnaire-short form; SEE, self-efficacy for exercise scale; PHQ-9, patient health questionnaire; GAD-7, generalized anxiety disorder 7-item scale; SSRS, social support rating scale.

between Class-3 (Low-baseline Slow-loss) and Class-4 (High-baseline Slow-loss) except for alcohol consumption and dietary self-efficacy. According to Tables 4 and 5, a higher male ratio was present in Class-1 and Class-2, and the differences in gender were highly effective in multiple subgroups, as compared with Class-3 and Class4. As regard the working status, Class- 2 included patients with the highest proportion of employment, while Class-3 was the only class with more non-incumbents than incumbents. As regard alcohol consumption, the proportion of drinkers in Class-1, Class-2, and Class-4 was significantly lower than that in Class-3, and Class-2 did not have any drinker.
As regard the psychological variables, dietary selfefficacy and exercise self-efficacy had higher effects than depression and social support according to the pairwise comparison. The level of dietary self-efficacy in the Class1, Class-2 and Class-3 was significantly higher than the level in the Class-4, Class-1 also had a statistically higher WEL-SF score than Class-3, and these differences were highly effective. Moreover, the order of dietary selfefficacy of each class was consistent with the order of $\mathrm{BF} \%$ at $12^{\text {th }}$ month. The distribution of the differences in patients' exercise self-efficacy among different classes was similar to the distribution of the differences in gender. The level of exercise self-efficacy in Class-1 and Class-2 was 
Table 5 Pairwise Comparison of Specific Variables

\begin{tabular}{|c|c|c|c|c|c|c|c|c|c|c|c|c|}
\hline \multirow[t]{3}{*}{ Variables } & \multicolumn{12}{|c|}{ Classifications of Pairwise Comparison } \\
\hline & \multicolumn{2}{|c|}{ ClvsC2 } & \multicolumn{2}{|c|}{ ClvsC3 } & \multicolumn{2}{|c|}{ ClvsC4 } & \multicolumn{2}{|c|}{$\mathrm{C} 2 \mathrm{vsC} 3$} & \multicolumn{2}{|c|}{$\mathrm{C} 2 \mathrm{vsC} 4$} & \multicolumn{2}{|c|}{ C3vsC4 } \\
\hline & $\boldsymbol{P}$ & $|\mathbf{d}|$ & $P$ & $|d|$ & $P$ & $|d|$ & $\mathbf{P}$ & $|\mathbf{d}|$ & $P$ & $|d|$ & $\mathbf{P}$ & $|\mathbf{d}|$ \\
\hline Gender & 0.777 & 0.03 & $<0.001 *$ & $0.56^{++}$ & $<0.001 *$ & $0.52^{++}$ & $<0.001 *$ & $0.52^{++}$ & $<0.001 *$ & $0.42^{+}$ & 0.765 & 0.02 \\
\hline $\begin{array}{l}\text { Working } \\
\text { status }\end{array}$ & 0.125 & 0.20 & 0.145 & 0.16 & 0.918 & 0.01 & 0.004 & $0.36^{+}$ & 0.063 & 0.14 & 0.055 & 0.14 \\
\hline $\begin{array}{l}\text { Alcohol } \\
\text { consumption }\end{array}$ & 0.221 & 0.14 & $0.001 *$ & $0.35^{+}$ & 0.091 & 0.12 & 0.001 & $0.40^{+}$ & 0.550 & 0.05 & $<0.00 I^{*}$ & $0.47^{+}$ \\
\hline $\begin{array}{l}\text { WEL-SF } \\
\text { score }\end{array}$ & 0.265 & 0.32 & $0.008^{*}$ & $0.63^{+}$ & $<0.001 *$ & $1.60^{++}$ & 0.248 & 0.36 & $<0.001 *$ & $1.41^{++}$ & $<0.001 *$ & $1.06^{++}$ \\
\hline SEE score & 0.369 & 0.21 & $<0.001 *$ & $1.54^{++}$ & $<0.001^{*}$ & $1.37^{++}$ & $<0.001 *$ & $1.86^{++}$ & $<0.001 *$ & $1.70^{++}$ & 0.134 & 0.26 \\
\hline $\begin{array}{l}\text { PHQ-9 } \\
\text { score }\end{array}$ & $0.07 \mid$ & 0.42 & 0.621 & 0.13 & $0.012 *$ & 0.43 & 0.193 & 0.31 & 0.882 & 0.03 & 0.097 & 0.31 \\
\hline SSRS score & 0.590 & 0.10 & 0.427 & 0.15 & $0.003 *$ & 0.44 & 0.243 & 0.27 & $0.005 *$ & $0.58^{+}$ & 0.084 & 0.34 \\
\hline
\end{tabular}

Notes: $|d|=$ Cohen's-d, indicating the effect size of difference. Bold ${ }^{+}$: medium effect (Continuous variable is $>0.50$, categorical variable is $>0.30$ ), Bold ${ }^{++}$: high effect (Continuous variable is $>0.80$, categorical variable is $>0.50$ ). Bold*: $P<0.05$.

Abbreviations: CI, Class-I; C2, Class-2; C3, Class-3; C4, Class-4; WEL-SF, weight efficacy lifestyle questionnaire-short form; SEE, self-efficacy for exercise scale; PHQ-9, patient health questionnaire; SSRS, social support rating scale.

significantly higher than the level in Class-3 and Class-4; all the differences had high effects. The difference in exercise self-efficacy between Class- 2 and Class-3 was the most significant, with an effective value of 1.86 . A certain relationship between levels of exercise selfefficacy and decline speed of BF\% was observed comparing the trajectory figure The higher the level of exercise self-efficacy, the faster the decrease of $\mathrm{BF} \%$. As regard depression, only Class-4 had a significantly higher depression level than Class-1. Furthermore, the depression level of Class- 1 and Class-3 was relatively low, while that of Class-2 and Class- 4 was high. Class- 1 and Class-2 obtained a significantly more social support than Class-4.

\section{Discussion}

The construction of the trajectory model is an appropriate approach to identify patients subjected to bariatric surgery with unsatisfactory body fat loss. In this study, patients were divided into four trajectory classes (Class-1"Lowbaseline Fast-loss", Class-2 "High-baseline Fast-loss", Class-3 "Low-baseline Slow-loss", Class-4 "Highbaseline Slow-loss"), and the results revealed that patients in Class-3 and Class-4 had not-ideal body fat loss trajectories within one year. BF\% among trajectory classes was significantly different, while the difference in baseline BMI was not significant. Therefore, $\mathrm{BF} \%$ could be used as an effective indicator to identify differences in body composition among post-bariatric patients with similar $\mathrm{BMI}$. $\mathrm{BF} \%$ is highly correlated with $\mathrm{BMI}$, but it is more accurate in identifying the individual metabolic risk, especially in patients with high or low muscle reserves. ${ }^{10}$ In China, some athletes undergo bariatric surgery to combat obesity after retirement. They have a large amount of muscle mass and correspondingly higher $\mathrm{BMI}$, then $\mathrm{BF} \%$ can better reflect their actual obesity situation than BMI. On the other hand, some patients focus only on their weight and lose muscle rapidly. They achieved BMI in the non-obese range but still had high metabolic risk after surgery. ${ }^{20}$ Although $\mathrm{BF} \%$ is not the preferred indicator compared with BMI, our goal in this work was to reflect the value of $\mathrm{BF} \%$ as an outcome indicator of bariatric surgery.

In addition, a declining trend of the $\mathrm{BF} \%$ trajectories was found, indicating that SG effectively reduced patients' weight and body fat in the first year after surgery. However, $55.1 \%$ of the patients belonged to the worst trajectory (Class-4), suggesting that many patients achieved little improvement in $\mathrm{BF} \%$. The difference in $\mathrm{BF} \%$ was due to the differences in diet, exercise and other factors in the lifestyles after surgery, and patients' psychosocial factors can also influence their lifestyle. ${ }^{21,22}$ Therefore, the early identification of patients who will have poor $\mathrm{BF} \%$ outcomes based on psychosocial factors is particularly important.

The difference in gender among different classes was significant. Males were predominantly present in the two classes (Class-1 and Class-2) with better trajectory classes, while the proportion of females in the classes with worse 
trajectory classes was very high. Thus, females could be considered as predictors of poor $\mathrm{BF} \%$ one year after surgery. Gender differences in fat metabolism could be the reasons why $\mathrm{BF} \%$ in female is higher and difficult to reduce. The basal fat oxidation is lower in females as compared to males, thereby contributing to a higher fat storage in women. ${ }^{23}$ Although the female normal range of $\mathrm{BF} \%$ is originally higher than the one of males, the difference in $\mathrm{BF} \%$ among classes was much larger than the difference in the normal range of $\mathrm{BF} \%$ between men and women, and the trajectories of Class- 3 and Class- 4 decreased more slowly. Indeed, gender is not only an important predictor of $\mathrm{BF} \%$ outcomes, but it may also affect the interclass distribution of several other variables. However, the subgroup analysis of the genders was not performed in this study because of the smaller sample size that might bring a negative impact on the efficacy of the growth mixture model. Thus, this limitation should be explored in the future.

Patients' baseline dietary self-efficacy and exercise selfefficacy were other two important predictors, and the pairwise comparisons revealed that these variables also resulted in high effects. The corresponding relationship between BF\% trajectories and the patients' dietary self-efficacy further emphasized that the eating behavior mainly affected the final $\mathrm{BF} \%$. This is because SG changes the physiological function and morphological structure of patient's stomach, thus patient's eating habits change enormously. ${ }^{24}$ However, the gradual recovery in the tolerance of specific food several months after surgery results in a change of habits by patients with low dietary self-efficacy who began to eat high-fat and high-sugar foods, ${ }^{25}$ which could promote the accumulation of fat mass and threat the weight control. ${ }^{26}$ Therefore, it is of utmost importance to perform health education to maintain a healthy diet in patients with low dietary self-efficacy. This finding is also in accordance with studies that focused on other bariatric surgery procedures. ${ }^{27}$ As regard exercise selfefficacy, a certain relationship between the confidence of overcoming the difficulties to perform physical exercise and the decline speed of $\mathrm{BF} \%$ could be present. Exercise was another important factor in controlling energy balance except for diet. Patients often lose lean mass in body composition early after surgery due to the limited diet, ${ }^{28}$ but they can maintain or even increase muscle mass 3 months after surgery by performing aerobic-strength exercise. ${ }^{29}$ Muscle mass is related to the basal metabolic rate; the higher the basal metabolic rate, the faster the speed of fat metabolism. The basal metabolic rate can be increased by maintaining the lean mass, which can further enhance the effects of postoperative body fat loss. ${ }^{30}$ Thus, exercise can be a virtuous cycle of weight control for patients. Therefore, patients who want to lose weight faster should be encouraged to exercise more. Patients with low exercise self-efficacy prior to surgery should be warned that the refusal to exercise could significantly affect body fat loss.

In addition to the above-mentioned high-effect predictors, the levels of social support, depression, alcohol consumption, and working status might also help the early prediction of $\mathrm{BF} \%$ outcomes. Class-3 patients had a significantly higher rate of non-working and alcohol consumption at baseline than the other patients, and the most evident feature of Class-3 trajectory was the slowest rate in the decline of the $\mathrm{BF} \%$. This could be due to the fact that obese people who undergo bariatric surgery could be more likely to reach the target amount of exercise at work, while obese non-workers have less activity, thus reducing body fat burning. ${ }^{31}$ The gratifying aspect for the patients subjected to bariatric surgery is that this surgery allowed many of them to go back to work, further promoting their body fat loss. ${ }^{32}$ Our result on alcohol consumption was consistent with a 3-year follow-up study, ${ }^{33}$ which revealed that preoperative drinking is a predictor of postoperative drinking in bariatric surgery patients, causing poor BMI decline. Furthermore, a large sample cross-sectional study found that alcoholism is a risk factor for poor eating habits. ${ }^{34}$ All these results suggest that drinking must first be stopped to help patients in having a diet regimen effective in controlling their weight.

A total of $36.0 \%(n=96)$ of the patients in this study had mild depression (PHQ-9 score $>4$ ), suggesting that depression in severely obese people was serious, and patients with relatively severe depression had higher BF $\%$ at baseline. Differences in PHQ-9 scores among classes may be determined using the patient's baseline $\mathrm{BF} \%$ since severe obesity induce depression. ${ }^{35}$ Patients with high baseline depression had high $\mathrm{BF} \%$ at $12^{\text {th }}$ month, and the difference was statistically significant. Therefore, it was possible to conclude that the high depression at baseline predicted poor $\mathrm{BF} \%$ outcome, although the predictive ability of this index might be weak. In addition, patients with significantly more social support achieved a lower BF $\%$ after one year, in agreement with previous evidence suggesting that good social support is beneficial to health. ${ }^{36}$ This is also important for bariatric surgery patients although the effect of social support was not high. Our finding was also in agreement with Conceião's study, ${ }^{37}$ which suggested that high levels of social support 
on patients' prior surgery lead to less depression, a reduced poor eating habits and better weight loss after surgery. Therefore, the hospital, family and society should work together to provide mental and psychological support to patients to ensure the success of bariatric surgery. ${ }^{38}$

Among the above-mentioned variables, gender, dietary self-efficacy and exercise self-efficacy have gained the attention of many studies. ${ }^{39}$ Diet and exercise are also routinely managed during the follow-up. ${ }^{40}$ Thus, the significance of other variables was more worthy of concerning. After comparing the overall trends of trajectories, the results showed that both Class- 1 and Class- 3 had a relative lower baseline $\mathrm{BF} \%$. These two classes were also characterized by low depression and good social support, and a significant difference in alcohol consumption was found between these classes. Besides, patients in Class- 2 and Class-4 had high baseline $\mathrm{BF} \%$, and a significant difference in social support was found between them. These findings suggested that patients with low baseline obesity had favorable psychosocial status, and lifestyle behaviors might be the main factor to predict their body fat loss and metabolic risk reduction, ${ }^{41}$ as well as the main factor to focus on during rehabilitation, whereas interpersonal factors such as social support might also determine body fat loss in severely obese patients. ${ }^{42}$ Further studies should investigate the differences in factors affecting weight loss among bariatric patients with varying degree of obesity.

Previous studies used physiological indicators to predict weight loss after bariatric surgery, ${ }^{43,44}$ thus having limitations although physiological indicators are more precise and quantifiable. The physiological factors are inconvenient to measure, and many of them are irreformable. On the contrary, psychosocial factors reflect patient's lifestyles and they are easier to monitor. Based on the predicator level, the postoperative behavior management could be easier by simply assessing the psychosocial status to identify patients who are more likely to have adverse weight loss outcomes. Patients may also feel that they are receiving more humane care during this process. ${ }^{45}$ Moreover, other studies on bariatric surgery explored the predictive effects of non-physiological factors on weight loss outcomes. ${ }^{46,47}$ Our results were not entirely consistent with these findings except for diet self-efficacy and depression probably because of the differences in surgical methods and lifestyle due to race. More importantly, our research classified the patients based on the trajectory model. The perspective focused on patient's body fat loss trends, which was different from the approach used in other studies, giving a comprehensive understanding on the longitudinal effects of bariatric surgery. In addition to examine whether patients' weight is in the non-obese range during the follow-up, the trajectories of weight loss and $\mathrm{BF} \%$ reduction should be also monitored. Focusing on weight at a single point obscured bariatric patients' longterm efforts to lose weight. Our hope was to present the postoperative weight loss achievement of patients more accurately through $\mathrm{BF} \%$ and trajectories.

The advantage of this study is that one year $\mathrm{BF} \%$ trajectory of Chinese SG patients was analyzed by a growth mixture model, identifying several classes of patients with different postoperative body fat loss effects. Their psychosocial data at baseline were compared according to classifications, thus showing the baseline characteristics of patients with poor body fat loss, helping the identification of these patients in clinical practice. As far as we know, this study was the first that constructed trajectories of post-bariatric patients in terms of $\mathrm{BF} \%$, and was also the first performed in Chinese SG patients. However, this work also presents some limitations. First, only SG patients were included. Although SG is the most widely performed bariatric surgery in China, ${ }^{48}$ studies exploring the trajectories of $\mathrm{BF} \%$ in other procedures such as RYGB and SG plus are needed in the future. Second, the baseline predictors were analyzed by differences and effects among trajectory classes. However, the relationship among predictors and $\mathrm{BF} \%$ still need further evaluation using regression analysis after collecting complete data during the whole follow-up period. This aspect is challenging because the organization of the postoperative follow-up in Chinese bariatric centers still needs improvements. Third, the tools used for the measurement of the factors have their limitations. Patient's diet and exercise behaviors were not directly measured, but the corresponding self-efficacy scale was used for psychological evaluation. The patient's BF\% was measured using a multi-frequency impedance tool (InBody) instead of using DEXA. InBody has the advantage of convenience, and is accurate for measuring total $\mathrm{BF} \%$ but not regional $\mathrm{BF} \%{ }^{49}$ However, DEXA is the best method to analyze body composition. ${ }^{50}$ Finally, the follow up of the $\mathrm{BF} \%$ in this study was one year, thus, a longer observation period is necessary to evaluate whether the trajectories' would continue with the same trend.

\section{Conclusion}

In conclusion, the predictors for poor $\mathrm{BF} \%$ trajectories in $\mathrm{SG}$ patients were female gender, low dietary self-efficacy, low exercise self-efficacy, non-working status, drinking habits, 
severe depression and low level of social support. Differences in gender, dietary self-efficacy and exercise self-efficacy had high effects. The lifestyle predicted body fat loss in low baseline obese patients, while body fat loss in patients with high baseline obesity could be influenced by the social support status. Healthcare professionals should early identify patients who tend to achieve not-ideal body fat loss outcomes based on the above predictors, thus helping them in strengthening the management of the behavior of these patients. Further studies should examine the predictive ability of these factors. These findings might potentially help the improvement of the body fat loss efficiency of post-bariatric patients.

\section{Data Sharing Statement}

All data generated or analyzed during this study are included in this article and its Supplementary Materials. Further enquiries can be directed to the corresponding author.

\section{Statement of Ethics}

The ethics review committee of the First Affiliated Hospital of Nanjing Medical University (2021-SR-031) approved this study. All patients who accept to participate signed the informed consent.

\section{Author Contributions}

All authors made a significant contribution to the work reported, whether that is in the conception, study design, execution, acquisition of data, analysis and interpretation, or in all these areas; took part in drafting, revising or critically reviewing the article; gave final approval of the version to be published; have agreed on the journal to which the article has been submitted; and agree to be accountable for all aspects of the work.

\section{Funding}

This work was supported by the Project "The exploration of trajectories and intervention program of frailty for gastric cancer survivors based on the health ecology theory" supported by National Natural Science Foundation of China [grant numbers 82073407]; the Project of "Design of intervention project for health behavior change of bariatric patients based on CCAM model" Funded by the Postgraduate Research \& Practice Innovation Program of Jiangsu Province [grant numbers KYCX20_1446]; the Priority Discipline Development Program of Jiangsu Higher Education Institutions(General Office, the People Government of Jiangsu Province (2018) No.87); and Project Early warning and intervention of childhood and adolescent obesity in the context of big data: Development and demonstrative application of iNATURE intelligent platform based on hospital, community, and family linkage supported by the Social Development Fund of Jiangsu Province (No: BE2021722).

\section{Disclosure}

The authors declare that they have no conflicts of interest.

\section{References}

1. Foreman KJ, Marquez N, Dolgert A, et al. Forecasting life expectancy, years of life lost, and all-cause and cause-specific mortality for 250 causes of death: reference and alternative scenarios for 2016-40 for 195 countries and territories. Lancet. 2018;392 (10159):2052-2090. doi:10.1016/S0140-6736(18)31694-5

2. Wang X, Chang XS, Gao L, et al. Effectiveness of laparoscopic sleeve gastrectomy for weight loss and obesity-associated co-morbidities: a 3-year outcome from Mainland Chinese patients. Surg Obes Relat Dis. 2016;12(7):1305-1311. doi:10.1016/j.soard.2016.03.004

3. King WC, Hinerman AS, Belle SH, Wahed AS, Courcoulas AP. Comparison of the performance of common measures of weight regain after bariatric surgery for association with clinical outcomes. JAMA. 2018;320(15):1560-1569. doi:10.1001/jama.2018.14433

4. Toh BC, Chan WH, Eng AKH, et al. Five-year long-term clinical outcome after bariatric metabolic surgery: a multi-ethnic Asian population in Singapore. Diabetes Obes Metab. 2018;20(7):1762-1765. doi: $10.1111 /$ dom. 13263

5. Nedeljkovic-Arsenovic O, Banovic M, Radenkovic D, et al. The amount of weight loss six months after bariatric surgery: it makes a difference. Obes Facts. 2019;12(3):281-290. doi:10.1159/000499387

6. Hindle A, de la Piedad Garcia X, Brennan L. Early post-operative psychosocial and weight predictors of later outcome in bariatric surgery: a systematic literature review. Obes Rev. 2017;18 (3):317-334. doi:10.1111/obr.12496

7. Sheets CS, Peat CM, Berg KC, et al. Post-operative psychosocial predictors of outcome in bariatric surgery. Obes Surg. 2015;25 (2):330-345. doi:10.1007/s11695-014-1490-9

8. Haghighat N, Ashtari-Larky D, Aghakhani L, et al. How does fat mass change in the first year after bariatric surgery? A systemic review and meta-analysis. Obes Surg. 2021;31(8):3799-3821. doi:10.1007/s11695-021-05512-9

9. Gómez-Ambrosi J, Andrada P, Valentí V, et al. Dissociation of body mass index, excess weight loss and body fat percentage trajectories after 3 years of gastric bypass: relationship with metabolic outcomes. Int J Obes (Lond). 2017;41(9):1379-1387. doi:10.1038/ijo.2017.134

10. Eriksson Hogling D, Bäckdahl J, Thorell A, Rydén M, Andersson DP. Metabolic impact of body fat percentage independent of body mass index in women with obesity remission after gastric bypass. Obes Surg. 2020;30(3):1086-1092. doi:10.1007/s11695-019-04304-6

11. Courcoulas AP, King WC, Belle SH, et al. Seven-year weight trajectories and health outcomes in the longitudinal assessment of bariatric surgery (LABS) study. JAMA Surg. 2018;153(5):427-434. doi:10.1001/jamasurg.2017.5025

12. Davis JA, Saunders R. Impact of weight trajectory after bariatric surgery on co-morbidity evolution and burden. BMC Health Serv Res. 2020;20(1):278. doi:10.1186/s12913-020-5042-9

13. Lee DH, Park KS, Ahn S, et al. Comparison of abdominal visceral adipose tissue area measured by computed tomography with that estimated by bioelectrical impedance analysis method in Korean subjects. Nutrients. 2015;7(12):10513-10524. doi:10.3390/ nu7125548 
14. Ames GE, Heckman MG, Grothe KB, Clark MM. Eating self-efficacy: development of a short-form WEL. Eat Behav. 2012;13(4):375-378. doi:10.1016/j.eatbeh.2012.03.013

15. Lee LL, Perng SJ, Ho CC, Hsu HM, Lau SC, Arthur A. A preliminary reliability and validity study of the Chinese version of the self-efficacy for exercise scale for older adults. Int J Nurs Stud. 2009;46(2):230-238. doi:10.1016/j.ijnurstu.2008.09.003

16. Wang W, Bian Q, Zhao Y, et al. Reliability and validity of the Chinese version of the Patient Health Questionnaire (PHQ-9) in the general population. Gen Hosp Psychiatry. 2014;36(5):539-544. doi:10.1016/j.genhosppsych.2014.05.021

17. He XY, Li CB, Qian J, Cui HS, Wu WY. Reliability and validity of a generalized anxiety disorder scale in general hospital outpatients. Shanghai Arch Psychiatry. 2010;22(4):200-203.

18. Liu JW, Li FY, Lian Y. Reliability and validity of social support rating scale. J Xinjiang Med Univ. 2008;Jan:4-6.

19. Lewis AJ, Sae-Koew JH, Toumbourou JW, Rowland B. Gender differences in trajectories of depressive symptoms across childhood and adolescence: a multi-group growth mixture model. J Affect Disord. 2020;Jan(260):463-472. doi:10.1016/j.jad.2019.09.027

20. De Lorenzo A, Bianchi A, Maroni P, et al. Adiposity rather than BMI determines metabolic risk. Int $J$ Cardiol. 2013;166(1):111-117. doi:10.1016/j.ijcard.2011.10.006

21. Köhler H, Markov V, Watschke A, et al. Psychosocial predictors of work ability in morbidly obese patients: results of a cross-sectional study in the context of bariatric surgery. Obes Facts. 2021;14 (1):56-63. doi:10.1159/000511735

22. Zalesin KC, Franklin BA, Lillystone MA, et al. Differential loss of fat and lean mass in the morbidly obese after bariatric surgery. Metab Syndr Relat Disord. 2010;8(1):15-20. doi:10.1089/met.2009.0012

23. Blaak E. Gender differences in fat metabolism. Curr Opin Clin Nutr Metab Care. 2001;4(6):499-502. doi:10.1097/00075197-20011100000006

24. Iossa A, Coluzzi I, Giannetta IB, Silecchia G. Weight loss and eating pattern 7 years after sleeve gastrectomy: experience of a bariatric center of excellence. Obes Surg. 2020;30(10):3747-3752. doi:10.1007/s11695-020-04699-7

25. Diaz-Lara C, Curtis C, Romero M, et al. Tolerance to specific foods after laparoscopic sleeve gastrectomy. Obes Surg. 2020;30 (10):3891-3897. doi:10.1007/s11695-020-04732-9

26. Coluzzi I, Raparelli L, Guarnacci L, et al. Food intake and changes in eating behavior after laparoscopic sleeve gastrectomy. Obes Surg. 2016;26(9):2059-2067. doi:10.1007/s11695-015-2043-6

27. Sysko R, Devlin MJ, Hildebrandt TB, Brewer SK, Zitsman JL, Walsh BT. Psychological outcomes and predictors of initial weight loss outcomes among severely obese adolescents receiving laparoscopic adjustable gastric banding. J Clin Psychiatry. 2012;73 (10):1351-1357. doi:10.4088/JCP.12m07690

28. Basciani S, Costantini D, Contini S, et al. Safety and efficacy of a multiphase dietetic protocol with meal replacements including a step with very low calorie diet. Endocrine. 2015;48(3):863-870. doi:10.1007/s12020-014-0355-2

29. Ren ZQ, Lu GD, Zhang TZ, Xu Q. Effect of physical exercise on weight loss and physical function following bariatric surgery: a meta-analysis of randomised controlled trials. BMJ Open. 2018;8 (10):e023208. doi:10.1136/bmjopen-2018-023208

30. Carey DG, Pliego GJ, Raymond RL. Body composition and metabolic changes following bariatric surgery: effects on fat mass, lean mass and basal metabolic rate: six months to one-year follow-up. Obes Surg. 2006;16(12):1602-1608. doi:10.1381/096089206779319347

31. Jiménez JM, García S, Carbajo MÁ, et al. Implications of professional occupation related to obesity in patients undergoing bariatric surgery. Int J Environ Res Public Health. 2020;17(15):5557. doi:10.3390/ijerph17155557
32. Courtney MJ, Mahawar K, Burnell P, et al. Occupational outcomes of obesity surgery-do the employed return to work, and do the unemployed find work? Obes Surg. 2018;28(4):963-969. doi:10.1007/ s11695-017-2963-4

33. Miller-Matero LR, Hamann A, LaLonde L, et al. Predictors of alcohol use after bariatric surgery. J Clin Psychol Med Settings. 2020;17:1-7.

34. Patino-Alonso MC, Recio-Rodríguez JI, Belio JF, et al. Factors associated with adherence to the Mediterranean diet in the adult population. J Acad Nutr Diet. 2014;114(4):583-589. doi:10.1016/j. jand.2013.07.038

35. Lin HY, Huang CK, Tai CM, et al. Psychiatric disorders of patients seeking obesity treatment. BMC Psychiatry. 2013;13:1. doi:10.1186/ 1471-244X-13-1

36. Kim S, Thomas PA. Direct and indirect pathways from social support to health? J Gerontol B Psychol Sci Soc Sci. 2019;74(6):1072-1080. doi:10.1093/geronb/gbx084

37. Conceição EM, Fernandes M, de Lourdes M, Pinto-Bastos A, Vaz AR, Ramalho S. Perceived social support before and after bariatric surgery: association with depression, problematic eating behaviors, and weight outcomes. Eat Weight Disord. 2020;25(3):679-692. doi:10.1007/s40519-019-00671-2

38. van Hout G, van Heck G. Bariatric psychology, psychological aspects of weight loss surgery. Obes Facts. 2009;2(1):10-15. doi:10.1159/ 000193564

39. Flølo TN, Tell GS, Kolotkin RL, et al. Eating self-efficacy as predictor of long-term weight loss and obesity-specific quality of life after sleeve gastrectomy: a prospective cohort study. Surg Obes Relat Dis. 2019;15(2):161-167. doi:10.1016/j.soard.2018.12.011

40. Mechanick JI, Apovian C, Brethauer S, et al. Clinical practice guidelines for the perioperative nutrition, metabolic, and nonsurgical support of patients undergoing bariatric procedures - 2019 update. Endocr Pract. 2019;25(12):1346-1359. doi:10.4158/GL-2019-0406

41. Varban OA, Bonham AJ, Finks JF, Telem DA, Obeid NR, Ghaferi AA. Is it worth it? Determining the health benefits of sleeve gastrectomy in patients with a body mass index $<35 \mathrm{~kg} / \mathrm{m} 2$. Surg Obes Relat Dis. 2020;16(2):248-253. doi:10.1016/j. soard.2019.10.027

42. Funk LM, Grubber JM, McVay MA, Olsen MK, Yancy WS, Voils CI. Patient predictors of weight loss following a behavioral weight management intervention among US Veterans with severe obesity. Eat Weight Disord. 2018;23(5):587-595. doi:10.1007/s40519-017-0425-6

43. Lee YC, Lee WJ, Lin YC, et al. Obesity and the decision tree: predictors of sustained weight loss after bariatric surgery. Hepatogastroenterology. 2009;56(96):1745-1749.

44. Lee WJ, Hur KY, Lakadawala M, et al. Predicting success of metabolic surgery: age, body mass index, C-peptide, and duration score. Surg Obes Relat Dis. 2013;9(3):379-384. doi:10.1016/j. soard.2012.07.015

45. Shen SC, Lin HY, Huang CK, Yen YC. Adherence to psychiatric follow-up predicts 1-year BMI loss in gastric bypass surgery patients. Obes Surg. 2016;26(4):810-815. doi:10.1007/s11695-0151821-5

46. Saiki A, Kanai R, Nakamura S, et al. Impact of mental health background and nutrition intake on medium-term weight loss in Japanese patients undergoing laparoscopic sleeve gastrectomy. Obes Facts. 2020;13(4):371-383. doi:10.1159/000509342

47. Leombruni P, Pierò A, Dosio D, et al. Psychological predictors of outcome in vertical banded gastroplasty: a 6 months prospective pilot study. Obes Surg. 2007;17(7):941-948. doi:10.1007/s11695-0079173-4

48. Hans PK, Guan W, Lin S, Liang H. Long-term outcome of laparoscopic sleeve gastrectomy from a single center in mainland China. Asian J Surg. 2018;41(3):285-290. doi:10.1016/j.asjsur.2017.04.003 
49. Czeck MA, Raymond-Pope CJ, Prescott E, Bisch KL, Dengel DR. Body fat percent assessment between electrical impedance myography and dual X-ray absorptiometry. Am J Hum Biol. 2020;32(2): e23330. doi:10.1002/ajhb.23330
50. Nickerson BS, McLester CN, McLester JR, Kliszczewicz BM. Agreement between 2 segmental bioimpedance devices, BOD POD, and DXA in obese adults. $J$ Clin Densitom. 2020;23(1):138-148. doi:10.1016/j.jocd.2019.04.005

\section{Publish your work in this journal}

Diabetes, Metabolic Syndrome and Obesity: Targets and Therapy is an international, peer-reviewed open-access journal committed to the rapid publication of the latest laboratory and clinical findings in the fields of diabetes, metabolic syndrome and obesity research. Original research, review, case reports, hypothesis formation, expert opinion and commentaries are all considered for publication. The manuscript management system is completely online and includes a very quick and fair peer-review system, which is all easy to use. Visit http://www.dovepress.com/testimonials.php to read real quotes from published authors.

Submit your manuscript here: https://www.dovepress.com/diabetes-metabolic-syndrome-and-obesity-targets-and-therapy-journal 